\title{
Estrategias de las Instituciones de Educación Superior para la Integración de las Tecnología de la Información y la Comunicación y de la Innovación en los Procesos de Enseñanza. Un Estudio en el Distrito de Barranquilla, Colombia
}

\author{
Freddy V. Marín ${ }^{(1)}$, Alicia de J. Inciarte ${ }^{(1)}$, Hugo G. Hernández ${ }^{(2)}$ y Remedios C. Pitre ${ }^{(3)}$ \\ (1) Departamento de Humanidades, Universidad de la Costa, Atlántico - Colombia \\ (e-mail: fmarin1@cuc.edu.co; ainciart1@cuc.edu.co) \\ (2) Corporación Universitaria Latinoamérica, Atlántico - Colombia \\ (e-mail: hugoghernandezpalma@gmail.com) \\ (3) Universidad de la Guajira, La Guajira - Colombia (e-mail: rpitre@uniguajira.edu.co)
}

Recibido Abr. 6, 2017; Aceptado Jun. 20, 2017; Versión final Ago. 8, 2017, Publicado Dic. 2017

\begin{abstract}
Resumen
El artículo da cuenta de una investigación cuyo objetivo fue analizar la dinámica de las universidades de Barranquilla, Colombia, en torno a las estrategias de integración de TIC e Innovación en la enseñanza. EI estudio se efectuó bajo un enfoque cuantitativo, diseño descriptivo-explicativo-analítico, mediante la técnica de la encuesta. Se aplicó un cuestionario conformado por cuatro categorías de análisis. Dentro de los resultados se encontró que el $71 \%$ de las instituciones estudiadas no disponen de cursos de actualización para que los docentes se capaciten en TIC de manera continua. Se encuentra también que el $64 \%$ de las instituciones estudiadas no diseñan metodologías para la enseñanza y aprendizaje mediado por las TIC con la participación de los docentes. Se concluye que las instituciones de educación superior de Barranquilla requieren estrategias de integración y articulación de tecnologías innovadoras que fortalezcan la didáctica en sus procesos de enseñanza.
\end{abstract}

Palabras clave: TIC; innovación; educación superior; enseñanza; aprendizaje

\section{Strategies of Institutions of Higher Education for the Integration of Information and Communication Technologies and of Innovation in the Teaching Process. A Study in the District of Barranquilla, Colombia}

\begin{abstract}
The article reports the results of study done to analyze the dynamics of the universities of Barranquilla, Colombia, around the strategies of integration of Information and Communication Technologies (ICT) and of Innovation in university teaching. The study was carried out under a quantitative approach, using a descriptive-explanatory-analytical design, through the survey technique. A questionnaire including four categories of analysis was applied. The results show that $71 \%$ of the institutions studied do not have refresher courses for teachers to train on ICT on a continuous basis. It is also found that $64 \%$ of the institutions studied do not design methodologies for teaching and learning mediated by ICT with participation of teachers. It is concluded that higher education institutions of Barranquilla require strategies of integration and articulation of innovative technologies that strengthen didactics in their teaching processes.
\end{abstract}

Keywords: ICT; innovation; higher education; teaching; learning 


\section{INTRODUCCIÓN}

Durante las últimas décadas se han desarrollado significativas trasformaciones en el campo de la educación superior; aspectos como la reorientación de los patrones educativos, la adquisición de nuevas tecnologías y el desarrollo de modelos de aprendizaje basados en competencias, se han impulsado con la finalidad de afianzar los procesos de enseñanza-aprendizaje y, por ende, de formar profesionales integrales capaces de hacer frente de manera dinámica y holística a los diversos retos del mundo globalizado actual. López et all (2016), exponen que en los programas académicos universitarios, se generan competencias cuyo carácter integrador, transferible y multifuncional requieren diseños curriculares coordinados e interdisciplinares, donde se posibilite una educación integral con la inserción de metodologías activas e innovadoras que propicien el mejoramiento continuo de la calidad. Al respecto, Icarte y Labate (2016), enfatizan la necesidad de definir y aplicar prácticas para evaluar la consistencia interna del currículo, en correspondencia con los procesos de enseñanza y los perfiles profesionales; ello supone disponer condiciones de orden humano y material que propicien tal propósito.

Al respecto, uno de los cambios a los que se ha enfrentado la academia, de acuerdo con Sisto y López (2014), es la trasformación de los sistemas de financiamiento, los cuales, de gestiones benefactoras pasaron a disminuir sus aportes significativamente para apoyar la educación; esta dinámica tiene un impacto directo sobre la investigación y contribuye a disminuir las posibilidades de desarrollo estructural de las instituciones de educación superior (Bollag, 2011). Por tanto, es necesario que las universidades reconozcan la necesidad de modificar sus paradigmas y se esfuercen por ser más competitivas, hasta el punto de constituirse como entidades sostenibles que no solo nutren a los seres humanos de conocimiento básico, sino que, aportan al desarrollo económico y social del territorio, por medio de lo que Casani y Rodríguez (2015) enuncian como una retícula de naturaleza empresarial para favorecer la resolución de problemas contextuales.

Ahora bien, dentro de las estrategias que pueden ser llevadas a cabo por las instituciones de educación superior en aras de ser más competitivas y sostenibles, se encuentra la apuesta por la innovación orientada a la intervención significativa del ámbito social, que en lo educativo, a decir de Tünnermann (2011), implica la transformación profunda de las bases del sistema, considerando los principios de sostenibilidad, progresividad y continuidad, apuntalando el fortalecimiento científico - tecnológico de referente institucionalizado y fundamentado en lo axiológico como forma de expresar el desarrollo humano de los colectivos sociales. Para que lo anterior sea posible, las instituciones de educación superior deben, según Mora (2004), efectuar una contextualización real de las condiciones endógenas y exógenas que las permean, teniendo como punto de referencia los cambios en los modelos educativos impulsados a partir de la era del conocimiento, en la cual, se resalta el valor económico de la educación y el desarrollo tecnológico. Igualmente, las universidades, desde la perspectiva de Naser y Ramírez (2014) deben diseñar políticas educativas que respondan de manera efectiva a los requerimientos y necesidades propias del medio, para lo cual, es preciso que revisen y mejoren continuamente la forma en que gestionan las tecnologías de la información y la comunicación (TIC), puesto que estas se constituyen como el medio contemporáneo para lograr objetivos estratégicos.

La innovación debe estar articulada de manera consistente a la tecnología; al respecto autores como Smith et al (2008) y Sánchez et al (2016), la asocian a las rutinas organizativas concebidas como acciones habituales desarrolladas por los actores para la transformación, de forma tal que se pueda responder efectivamente a los desafíos del medio, mediante la tecnología e innovación desde una relación de complementariedad; ello posibilita los procesos de enseñanza-aprendizaje, además, permite a los futuros profesionales acceder a la información, no solo como sujetos pasivos, que se limitan a la reproducción de contenidos, sino como actores participativos que, de manera creativa desarrollan nuevas maneras de "saber" y de "saber hacer" en sus prácticas diarias, a partir del relacionamiento y la apertura al conocimiento que permiten las nuevas tecnologías y la versatilidad que imprime la innovación. La innovación en el marco de las TIC, además, incentiva el desarrollo del conocimiento propio (Belloch, 2012), abre nuevas vías de aprendizaje (Meneses, 2007) y potencia las habilidades, direccionado a los estudiantes universitarios ampliar su cosmovisión y capacidad crítica- reflexiva.

Empero, para que los futuros profesionales adquieran las competencias necesarias para aprovechar las ventajas ofrecidas por la innovación y las TIC, es preciso que las instituciones de educación superior desarrollen estrategias de integración que se orienten a la consolidación de procesos pedagógicos y de aprendizaje, las cuales permitan tanto a los docentes como a los estudiantes comprender el alcance de las TIC en la academia e identificar la importancia de la innovación como pilar del desarrollo integral, de este modo, se puedan formular desde las instituciones de educación superior proyectos de investigación o de aplicación cuyo impacto social, económico y cultural sea favorable para la sociedad. En el marco de estas consideraciones, Niebles et al (2016), destacan la necesidad de que las organizaciones educativas asuman 
con responsabilidad y compromiso la integración de las TIC al ciclo didáctico y a la gestión curricular, garantizando de esta forma mecanismos de innovación, autonomía y autoregulación en el proceso formativo.

La evolución de la situación problema, desde la perspectiva de Tapasco y Giraldo (2017), ha estado asociada al significado y carácter prioritario que los diferentes actores atribuyen a la innovación tecnológica en la educación superior; consecuentemente se derivan comportamientos y acciones que pueden representar agentes dinamizadores o inhibidores para la formación integral. Específicamente, en las instituciones de educación superior ubicadas en el distrito de Barranquilla, Colombia, se percibe completa autonomía para la gestión de sus procesos TIC y de innovación, es decir, no están obligadas a desarrollar programas de integración y proyectos de promoción de TIC específicos, al no ser un requisito fundamental para que adquieran su registro calificado y puedan operar; sin embargo, cuando se trata de obtener un certificado de acreditación en alta calidad, estas instituciones implementan programas y proyectos TIC pues, de este modo demuestran la apuesta que tienen en la nueva era del conocimiento y la capacidad de gestionar acciones vinculadas a la formación profesional con base a la innovación y las TIC.

No todas las universidades del distrito de Barranquilla, Colombia cuentan con la mejor infraestructura tecnológica; en muchos casos estas entidades adolecen de los recursos necesarios para automatizar sus procesos, proporcionar redes de internet potentes para sus estudiantes o dotar a las aulas de clase de herramientas tecnológicas que faciliten e impulsen los procesos de enseñanza-aprendizaje; vinculado a esta problemática se evidencia que "desde la perspectiva del presupuesto público nacional, sólo el 0,58\% de este se destina a actividades de Ciencia tecnología e innovación" (Colciencias, 2013), de allí que las actividades de investigación e integración tecnológica por parte de las universidades sean prácticamente nulas y que estas carezcan del apoyo estatal para mejorar de la manera adecuada su equipamiento científico y tecnológico, lo que las deja por fuera en un proceso de acreditación de alta calidad.

De lo anterior, la necesidad de que las instituciones de educación superior conciban estrategias para integrar las TIC y la innovación en sus procesos de enseñanza de una manera óptima y eficiente, con mínimo uso de recursos económicos y, por el contrario, impulsando la participación del capital humano universitario. A partir de estas estrategias puede tenerse un impacto positivo en la medida que se incrementa la capacidad de investigación, aumenta la promoción de la cultura de la innovación, y se impulsa la gestión de las TIC en este tipo de entidades. Consecuentemente, en el presente artículo se analiza el panorama actual de las universidades del distrito de Barranquilla, en torno a las estrategias de integración de TIC e Innovación en los procesos de enseñanza; si bien, en la actualidad existen diversas investigaciones sobre el tema (Sarkar, 2012; Vajargah et al. 2010; Oye, 2012; Cózar y Gutiérrez et al, 2016), en el caso específico del distrito de Barranquilla existen muy pocos estudios que documenten el comportamiento de las referidas variables.

\section{METODOLOGÍA}

La investigación se efectuó bajo un enfoque metodológico cuantitativo y tuvo un alcance descriptivoexplicativo - analítico; implicó un proceso de recolección de datos en las universidades del distrito de Barranquilla, Colombia, que posteriormente fueron contrastados a partir de un análisis correlacional. La muestra del estudio es de tipo no probabilístico y se planteó con base a criterios de accesibilidad; para ser determinada se seleccionó el universo poblacional, el cual corresponde a 18 instituciones de educación superior que en la actualidad ofrecen carreras profesionales; a partir de la identificación de las entidades, se procedió a la aplicación de la fórmula para determinar el tamaño de muestra para poblaciones finitas (Morales, 2008) según la ec. (1):

$n=\frac{N \cdot\left[0.5 . \alpha_{c}\right]}{1+(\mathrm{N}-1) * e^{2}}$

En esta ecuación $N$ es el tamaño poblacional (universo), $\alpha_{c}$ valor del nivel de confianza, $e$ es el margen de error. En la Tabla 1 se muestra que el nivel de confianza corresponde a un $90 \%$ y un margen de error del 10\% (selección desde la intencionalidad de los investigadores). Para este caso la muestra estudiada, según los resultados de la fórmula aplicada corresponde a 14 instituciones de educación superior.

El instrumento utilizado fue el Cuestionario cerrado, el cual constó de cuatro categorías de análisis: apropiación de tecnologías en el modelo educativo; producción de materiales digitales; formación de docentes para el uso significativo de las TIC; investigación en innovación y TIC; cada una de estas categorías es abordada mediante 5 ítems o preguntas, para un total de 20 reactivos. Para la recolección de la información se envió el cuestionario a un directivo o encargado de la gestión de TIC de cada universidad, previo consentimiento informado, en el cual se notificó a las universidades participantes del tratamiento 
efectivo de sus datos y de la confidencialidad del estudio, insistiendo en la importancia de que las preguntas fueran respondidas en su totalidad y en forma veraz, a partir de lo cual, se logró una tasa de respuesta del $100 \%$. Es necesario resaltar que el estudio se desarrolló teniendo en cuenta los principios éticos de la American Educational Research Association (2011), lo que garantizó la privacidad de las instituciones y de los encuestados; además, se obtuvo la autorización requerida para el cumplimiento del proceso. Así mismo, la recolección, tabulación e interpretación de los datos pretendió la objetividad desde un plano argumentativo.

Tabla 1: Características estadísticas de la aplicación de la fórmula para la determinación de la muestra

\begin{tabular}{|l|lll|}
\hline Margen de Error & $10,0 \%$ & & \\
\hline Tamaño población & $18^{*}$ & & \\
\hline Nivel de confianza & $90 \%{ }^{*}$ & & \\
\hline Valores Z (Valor nivel de confianza) & $90 \% \quad 95 \%$ & $97 \%$ \\
\hline Varianza (valor para reemplazar en la fórmula) & 1,645 & 1,960 & 2,170 \\
\hline
\end{tabular}

\section{RESULTADOS Y DISCUSIÓN}

La Fig. 1 muestra los resultados asociados a la primera categoría de estudio: "apropiación de tecnologías en el modelo educativo"; su abordaje fue mediante 5 preguntas, que se direccionaron principalmente a constatar la estructura de sustentabilidad de las instituciones de educación superior que favorecen la inserción de las TIC en el proceso didáctico.

¿La institución cuenta con una estructura organizativa de TIC e Innovación: unidades y equipos de apoyo?

¿La institución ha establecido en su PE. metodologías para la enseñanza y de aprendizaje mediado con TIC?

¿La institución posee políticas para la integración de tecnologías en los procesos educativos?

¿La institución tiene experiencias concretas de diseño e implementación de cursos con incorporación de TIC?

¿La institución posee la infraestructura tecnológica apropiada?

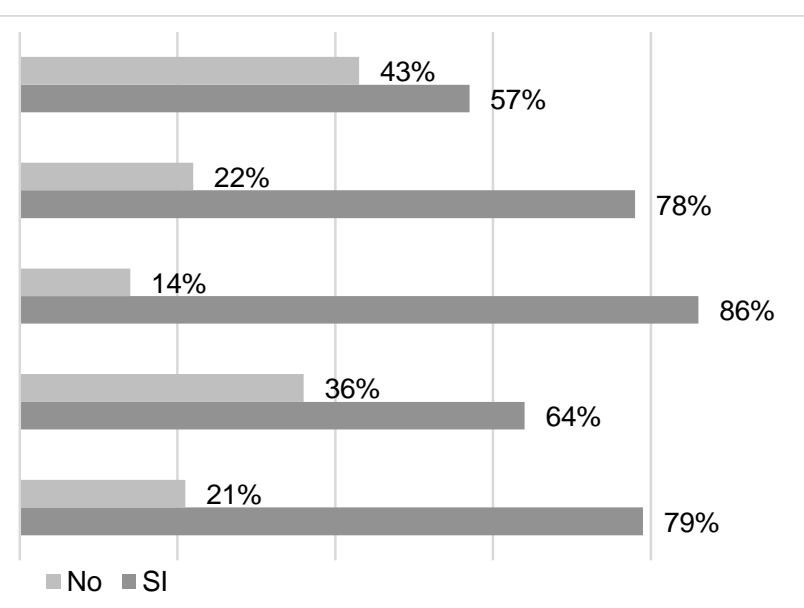

Fig. 1: Apropiación de tecnologías en el modelo educativo.

En relación con la primera pregunta, el 57\% de los participantes afirmó que la institución cuenta con una estructura organizativa de TIC e innovación integrada por unidades o grupos de apoyo, los cuales generalmente son conformados por docentes y algunos estudiantes; sin embargo, $43 \%$ restante asegura no contar con tal estructura organizativa. Cuando las universidades configuran grupos o unidades que se dedican principalmente a la gestión de los procesos TIC y de innovación, se pueden facilitar y mejorar continuamente sus proyectos. Bucceria et al (2014), asume una estructura de naturaleza matricial, con relaciones horizontales, donde confluye las fortalezas de los grupos de trabajo en función de sus competencias personales y profesionales. De igual modo, este tipo de estructuras posibilita la movilización de gran cantidad de intangibles y la creación de conocimiento tácito y explícito relacionado con las nuevas tecnologías y con la innovación para afianzar los procesos educativos (Arias y Aristizábal 2008).

En la segunda pregunta, el $78 \%$ de los participantes ofreció una postura favorable, argumentando que la institución ha establecido en su proyecto educativo (P.E) metodologías para la enseñanza y el aprendizaje mediado por las TIC; en contraste con el $22 \%$ restante que niega la incorporación de estrategias de esta naturaleza. Respecto a la tercera pregunta, el $86 \%$ de los participantes aseveró que en la actualidad la institución posee políticas de integración de las tecnologías a los procesos educativos; en contraste con el $14 \%$ quienes niegan esta afirmación. Estas dos preguntas se direccionan a establecer el grado de compromiso que las instituciones de educación superior objeto de estudio tienen en pro de la apropiación de las TIC; está claro que, si estas instituciones introducen metodologías TIC a su P.E y poseen políticas de 
integración respecto de la innovación, pueden satisfacer los intereses de los actores educativos y posibilitar la mediación didáctica - pedagógica (Zenteno y Mortera, 2011); al respecto, Nolasco y Ojeda (2016) y Sunkel et al (2014) resaltan la necesidad de que los sistemas educativos disminuyan la brecha en el acceso al mundo digital como alternativa válida para el fortalecimiento del desempeño académico, formación docente, cobertura y calidad educativa.

Cuando se preguntó a los participantes si la institución tiene experiencias concretas de diseño e implementación de cursos con incorporación de las TIC, el 64\% responde afirmativamente; caso contrario un $36 \%$ de las instituciones no han adelantado estos procesos; situación que puede representar un agente inhibidor del desarrollo organizacional. De igual modo, se consultó a los participantes si la institución posee la infraestructura tecnológica apropiada, el $79 \%$ expresó que si, en contraste con el $21 \%$ que piensa diferente. De acuerdo con Riveros et al (2014) y Barona et al (2010) una infraestructura tecnológica acorde con la gestión educativa, permite el acceso a la innovación, así como también el surgimiento de redes de comunicación, lo que representa en sí misma un proceso de mediación de los aprendizajes y el hacer científico - tecnológico.

En la Fig. 2, por su parte, se establecen los resultados de la segunda categoría de estudio: "producción de materiales digitales", la cual contenía 5 preguntas, orientadas a validar la cantidad, calidad y procesos instruccionales desarrollados para crear contenidos digitales, que contribuyan a la producción de nuevos conocimiento e innovaciones, en el marco de las TIC.

¿La institución incentiva a sus estudiantes a producir conocimiento para luego...

La institución gestiona de manera efectiva los derechos de autor de los materiales digitales..

¿La institución diseña o ha diseñado materiales educativos utilizando TIC?

¿La institución cuenta con recursos educativos digitales multimediales, interactivos y de fácil...

¿La institución cuenta con plataformas tecnológicas apropiadas para la producción...

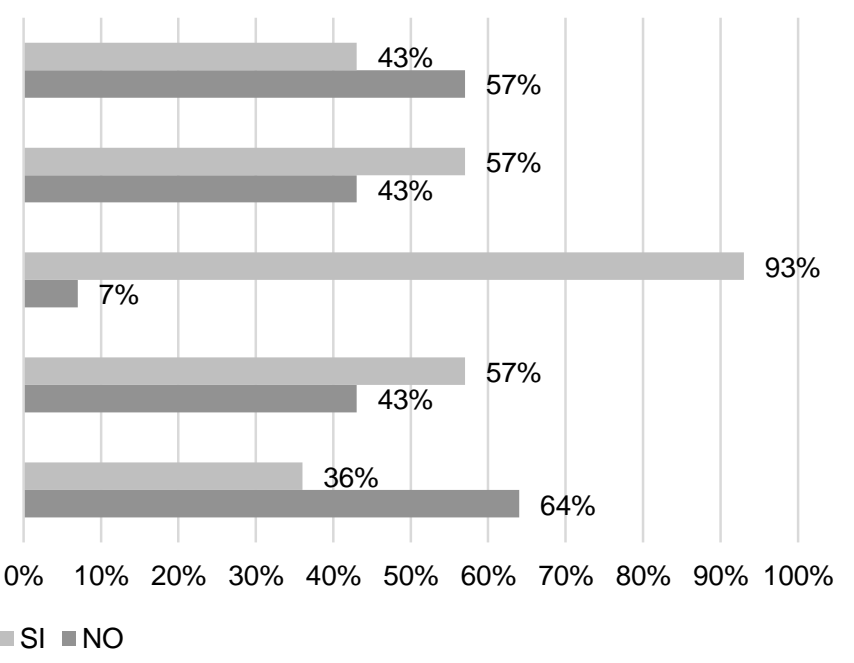

Fig. 2: Producción de materiales digitales

Cuando se consultó a los participantes si la institución de educación superior incentiva a sus estudiantes a producir conocimiento para luego digitalizarlo y ofrecerlo a la comunidad académica, solo el $43 \%$ respondió afirmativamente, mientras que un alto porcentaje (57\%) negó tal acción; estos hallazgos son preocupantes, puesto que, si desde la academia no se consolidan estrategias que permitan a los estudiantes contribuir a la creación de activos intangibles, difícilmente podría esperarse que la investigación en Colombia se posicione como un imperativo de desarrollo. Aunada a esta problemática, a partir del estudio se constata que solo el $57 \%$ de las instituciones gestionan de manera efectiva los derechos de autor de los materiales digitales de sus docentes y estudiantes; tal situación puede ser contraproducente y causar incluso problemas de tipo legal. (Martínez, 2006; Cárdenas, 2016).

De igual modo, se consultó si la institución diseña materiales educativos utilizando TIC; El 93\% responde favorablemente, lo que lleva a suponer que estas entidades se apoyan en las TIC para el desarrollo de sus procesos de enseñanza; sin embargo resulta contradictorio, que solo el $57 \%$ de las universidades cuente con recursos digitales mutimediales, interactivos, de fácil acceso y más aún, cuando el $64 \%$ afirma que en las instituciones no se dispone de plataformas tecnológicas apropiadas para la producción digital de contenidos. Estos hallazgos reflejan una ambivalencia, que supone la necesidad de fortalecer lineamientos de políticas donde se priorice la incorporación de las TIC, y en consecuencia se actúe aplicando estrategias para la consolidación de una infraestructura tecnológica de soporte, a procesos formativos integrales e innovadores. Igualmente, los resultados podrían develar que los docentes, conscientes de la necesidad por mejorar e innovar en sus prácticas pedagógicas, desarrollen iniciativas y esfuerzos particulares en atención a la creación de algunos materiales digitales. Tal como lo sostiene, Gómez y Montero (2015), debe concebirse la virtualidad como un componente necesario en los procesos formativos, desde una visión complementaria e integradora. 
En la Fig. 3, se exponen los resultados de la tercera categoría de estudio: "formación de docentes para uso significativo de las TIC", mediante 5 cuestionamientos se indagó acerca del apoyo que los docentes reciben de parte de las instituciones de educación superior para capacitarse y, aplicar estrategias fundamentadas en las TIC e innovación. A partir del análisis de los resultados de la categoría 3 , se perciben ciertas debilidades en las instituciones de educación superior objeto de estudio: El $71 \%$ de estas entidades no disponen de cursos de actualización para que los docentes se formen en TIC de manera continua, esta es una desventaja en la medida que pueden quedar al margen de los adelantos tecnológicos, siendo difícil identificar su impacto como agente de mediación para la construcción del conocimiento (Valdés et.al. 2011), de igual modo, se evidencia que el $64 \%$ de las instituciones estudiadas no han incorporado a los docentes al diseño de metodologías con base en las TIC; tampoco se ubican experiencias significativas en la formación de profesores en este ámbito del saber humano; se reitera la necesidad de orientar políticas de gestión en esta direccionalidad.

Aun cuando no se revelan políticas institucionales orientadas a la formación en TIC, llama la atención que el $64 \%$ de los docentes afirman utilizar estas herramientas en su práctica pedagógica; asimismo, un $43 \%$ de los sujetos coinciden en su participación activa en procesos de innovación asociados la inserción de las TIC en la práctica pedagógica y la mediación didáctica. Al igual que lo observado en la categoría: producción de medios digitales, se aprecian inconsistencias en el sentido de correspondencia entre la estrategia de crecimiento y desarrollo tecnológico definida por la institución, y la forma como los docentes ejecutan prácticas pedagógicas innovadoras sustentadas en las tecnologías de la información y comunicación. La tesis de la innovación en los procesos educativos en el contexto de las TIC es reforzada por autores como Maya y Qismullah, 2015; Ranjit y Chan, 2014; Ndibalema, 2014; Maracci y Mariotti, 2010, cuando asumen los entornos virtuales de aprendizaje como espacios dinámicos donde el estudiante desarrolla competencias científicas para la construcción de un aprendizaje significativo.

¿La institucion dispone de cursos de actualización para que los docentes se capaciten en TIC de manera...

$¿$ Los docentes suelen utilizar herramientas TIC como apoyo en su proceso de enseñanza?

$¿$ Los docentes participan de manera activa en los procesos relacionados con la innovación y las TIC?

¿La institución ha estructurado de la mano de los docentes metodologías para la enseñanza y de...

¿La institución ha tenido experiencias en formación de profesores en la integración de TIC en la educación?

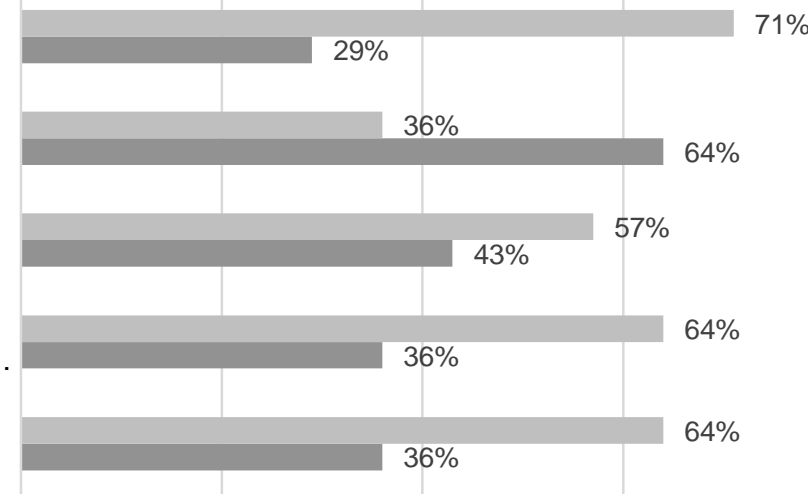

$\square \mathrm{NO} \backsim \mathrm{SI}$

Fig. 3: Formación de docentes para uso significativo de TIC

En la Fig. 4, se muestran los resultados de la cuarta categoría de estudio: "investigación relacionada con las TIC y la innovación", se consultó si las instituciones objeto de estudio adelantan proyectos de investigación para identificar el impacto, ventajas, limitaciones, en relación a la pertinencia de las TIC como agente dinamizador de la gestión didáctica, pedagógica y curricular. $¿$ Incluye a los estudiantes en la construcción de conocimiento
en torno a TIC e Innovación mediante procesos de trabajo colaborativo?

¿ La institución ha llevado a cabo procesos de innovación de la mano de aliados estratégicos?

¿La institución ha indagado acerca del impacto de las TIC en la formación de competencias en los estudiantes?

¿La institución ha indagado acerca del impacto de las TIC en las practicas pedagógicas?

¿La institución ha adelantado proyectos de investigación relacionados con las TIC y la Innovación?

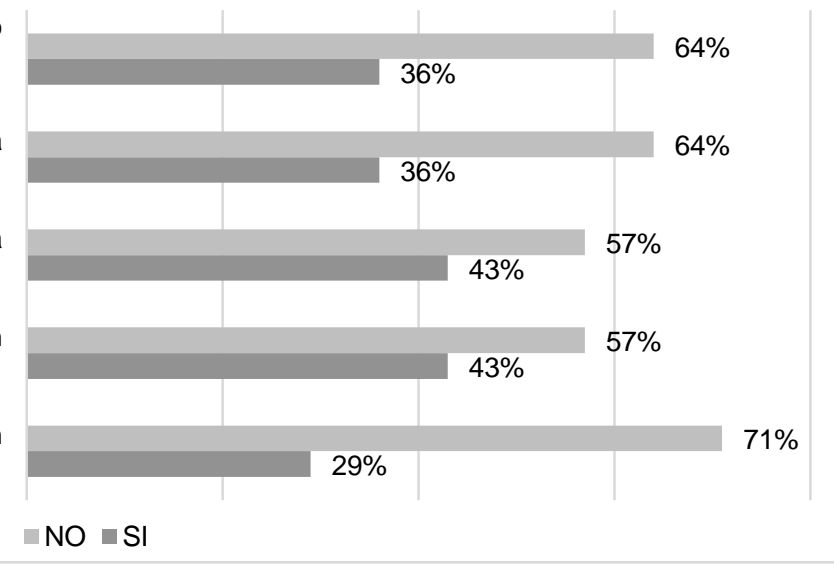

$\square \mathrm{NO} \backsim \mathrm{SI}$

Fig. 4: Investigación relacionada con las TIC y la Innovación 
El $64 \%$ de los participantes afirma que las instituciones de educación superior no incluyen a sus estudiantes en la construcción de conocimiento sobre TIC e Innovación, mediante procesos de trabajo colaborativo; se infieren restricciones impuestas para el acceso de los jóvenes, lo que puede resultar contradictorio si se considera lo expuesto por Prensky (2010) cuando asocia el desarrollo de potencialidades propias de la cultura tecnológica; por ejemplo las referidas a sus habilidades para el manejo de diferentes interfaces.

De igual forma, el estudio demuestra que solo el $36 \%$ de las instituciones han llevado a cabo procesos de innovación con fundamento en alianzas estratégicas ganar - ganar; deben generarse espacios para el fortalecimiento de relaciones universidad - gobierno - empresa con base en redes de cooperación científico técnica y la convergencia en clúster de apoyo al fortalecimiento de la política académica. A partir de lo anterior, se puede argumentar el por qué, solo el $43 \%$ de las instituciones hayan indagado acerca del impacto de las TIC en la formación de competencias en los estudiantes y que este mismo porcentaje haya investigado acerca del impacto de las TIC en las practicas pedagógicas; así pues, si las universidades no buscan y establecen alianzas estratégicas, además, si no trabajan de manera colaborativa con los actores educativos en lo relacionado con la promoción de la investigación, minimizan sus posibilidades de hacerse competitivas afectando su ciclo de vida.

\section{CONCLUSIONES}

Resulta impostergable el diseño de políticas y lineamientos de acción orientados al fortalecimiento de los distintos programas académicos, para contribuir de forma efectiva con la formación de profesionales integrales. La dinámica de las universidades de Barranquilla, Colombia requiere la definición y aplicación de estrategias de integración de las TIC e innovación en la mediación didáctica - pedagógica, para contribuir a que los estudiantes desarrollen un perfil de competencias científicas que orienten la búsqueda de información pertinente y la construcción de un conocimiento significativo, tomando en consideración la dimensión ética asociada principalmente al derecho de autor.

Los modelos educativos que fundamentan el currículo universitario deben considerar en forma explícita procesos de apropiación de la tecnología, así como también, la incorporación de la innovación a sus tareas fundamentales. Se requiere que el proyecto educativo institucionalice tales necesidades, mediante su integración como componentes fundamentales. Ello tiene implicaciones en la disponibilidad de condiciones físico - ambientales que coadyuven al logro del referido propósito, tal es el caso, de una adecuada infraestructura tecnológica de soporte.

Se concluye en la necesidad de que las instituciones de educación superior, enfaticen la formación del profesorado desde la mediación didáctica de las TIC, con la intención de fortalecer su práctica pedagógica e incidir en la cualificación del docente, asociada al desarrollo de capacidades para responder de forma ágil y oportuna a los desafíos propios de la era digital.

Finalmente, mediante el estudio se develó la necesidad de profundizar procesos investigativos cuya temática central sean las TIC e innovación en las instituciones de educación superior; surgen requerimientos orientados a que estas instituciones configuren comunidades y redes de aprendizaje donde se consoliden proyectos de base interdisciplinar, a través del trabajo colaborativo y en red, para integrar los diferentes actores que dinamizan la gestión académica universitaria; se pretende contribuir a la generación de conocimiento útil y socialmente pertinente, mediante la formación integral de los profesionales.

\section{REFERENCIAS}

American Educational Research Association, Ethical Standars of the American Educational Research Association (2011)

Arias-Pérez, J.E. y Aristizábal-Botero, C.A., Influencia de la Estructura Organizacional en la Creación de Conocimiento, Estudio del Caso EPM Medellín, Semestre Económico, 11(22), 161-184 (2008)

Barona Ríos, C., García Ponce de León, O. y Torres Velandia, S.A., Infraestructura Tecnológica y Apropiación de las TIC en la Universidad Autónoma del Estado de Morelos. Estudio de caso, Perfiles Educativos, 32(1), 105-127 (2010)

Belloch, C., Las Tecnologías de la Información y Comunicación en el Aprendizaje. (En línea: https://goo.gl/kDoBvC. Acceso: 4 de enero de 2017). Universidad de Valencia, España (2012)

Bollag, B., Grandes Cambios Sacuden la Educación Superior. Organización de las Naciones Unidas para la Educación la Ciencia y la Cultura. (En línea: https://goo.gl/eRuxuk. Acceso: 3 de enero de 2017) ESALC UNESCO (2011) 
Bucceria, M.G., Montenegroa, M.E. y Buccoloa, M.L., Estructura Organizacional del Área TIC en la Universidad de Buenos Aires: Desde Soporte Técnico hacia un Centro de Servicios para el Usuario. IV Conferencia de Directores de Tecnología de Información TICAL 2014, Gestión de las TICs para la Investigación y la Colaboración, Cancún (2014)

Cárdenas-Zardoni, H., La Compleja Relación entre la Biblioteca Universitaria y la Ley Federal de Derechos de Autor: Implicaciones y Responsabilidades en la Reproducción y Fotocopiado de Materiales, Biblioteca Universitaria, 19(1), 33-50 (2016)

Casani-Fernández de Navarrete, F. y Rodríguez Pomeda, J., Cambios y Tendencias en la Educación Superior: Ios Retos para la Universidad, Encuentros Multidisciplinares, 17(49), 1-10 (2015)

Colciencias. Plan Estratégico Departamental de Ciencia, Tecnología e Innovación del Atlántico. PEDCTI 2012 - 2022. Clúster Logística y Clúster Procesamiento de Productos de Molinería y Concentrados: Informe Final. (En línea: https://goo.gl/tBecDs. Acceso: 9 de enero de 2017) Barranquilla, Julio (2013)

Cózar-Gutiérrez, R., De Moya-Martínez, M.V. Hernández-Bravo, J.A. y Hernández-Bravo, J.R., Conocimiento y Uso de las Tecnologías de la Información y las Comunicaciones (TIC) según el Estilo de Aprendizaje de los Futuros Maestros, doi:10.4067/50718-520620160004000400003, Formación Universitaria (en línea), 9(6), 105-118 (2016)

Gómez, S.M. y Montero Caicedo, L., Estudiar Carreras Universitarias en Modalidades E- learning y Blearning, Revista Lasallista de Investigación, 12(2), 94-104 (2015)

Icarte, G.A. y Labate, H., Metodología para la Revisión y Actualización de un Diseño Curricular de una Carrera Universitaria Incorporando Conceptos de Aprendizaje Basado en Competencias, doi:10.4067/50718-520620160004000400003, Formación Universitaria (en línea), 9(2), 13-16 (2016)

López, C. Benedito, V. y León, M., El Enfoque de Competencias en la Formación Universitaria y su Impacto en la Evaluación. La Perspectiva de un Grupo de Profesionales Expertos en Pedagogía, doi:10.4067/50718520620160004000400003, Formación Universitaria (en línea), 9(4), 11-22 (2016)

Maracci, M. y Mariotti, M.A., The Teacher's Use of ICT Tools in the Classroomafter a Semiotic Mediation Approach, Proceedings of CERME, 1320-1329 (2010)

Martínez Rincones, J.F., Universidad, Propiedad Intelectual y Protección, Propiedad Intelectual, 5(1), 273$290(2006)$

Maya-Silviyanti, T. y Qismullah-Yusuf. Y., Efl Teachers' Perceptions on Using ICT In Their Teaching: To Use Or To Reject? Teaching English with Technology, 15(4), 29-43 (2015)

Meneses-Benítez, G., Las TICs en la Universidad. (En línea: https://goo.gl/qs3u79. Acceso: 4 de enero de 2017). ISBN:978-84-691-0359-3/DL: T.2183-2007, Universitat Rovira I Virgili (2007)

Mora, J.G., La Necesidad del Cambio Educativo para la Sociedad del Conocimiento, Revista Iberoamericana de Educación, 35(1), 13-37 (2004)

Morales Vallejo. P., Estadística Aplicada a las Ciencias Sociales, Universidad Pontificia Comillas, Madrid, España (2008)

Naser, A. y Ramírez, A., Plan de Gobierno Abierto. Una Hoja de Ruta para los Gobiernos de la Región. CEPAL (2014)

Ndibalema, P., Teachers' Attitudes Towards the Use of Information Communication Technology (ICT) as a Pedagogical Tool in Secondary Schools in Tanzania: The Case of Kondoa District, International Journal of Education and Research, 2(4), 1-16 (2014)

Niebles-Núñez, W.A., Hernández-Palma, H.G. y Cardona-Arbeláez, D.C., Gestión Tecnológica del Conocimiento: Herramienta Moderna para la Gerencia de Instituciones Educativas, Revista Investigación, Desarrollo, Innovación, 7(1), 25-36 (2016)

Nolasco-Vázquez, P. y Ojeda-Ramírez, M.M., La Evaluación de la Integración de las TIC en la Educación Superior: Fundamento para una Metodología, RED-Revista de Educación a Distancia, 9(48), 1-24 (2016) 
Oye, N. D., Shallsuku, Z.K. y lahad, A., The Role of ICT in Education: Focus on University Undergraduates taking Mathematics as a Course, International Journal of Advanced Computer Science and Applications: 3(2), 136-143 (2012)

Prensky, M., Nativos e Inmigrantes Digitales. (En línea: https://goo.gl/CG6ctU. Acceso: 2 de enero 2017). Institución Educativa SEK, Adaptación al castellano del texto original "Digital Natives, Digital Immigrants" (2010)

Ranjit, T.K. y Chan, S., Teacher Readiness on ICT Integration in Teaching-Learning: a Malaysian Case Study, International Journal of Asian Social Science, 4(7), 874-885 (2014)

Riveros, V., Miquilena, E. y Acosta, R., La Infraestructura de las Tecnologías de la Información y Comunicación como Mediadoras y el Aprendizaje de la Biología, Telos, 16(1), 11-30 (2014)

Sarkar, S., The Role of Information and Communication Technology (ICT) in Higher Education for the $21^{\text {st }}$ Century, The Science Probe, 1(1), 30-40 (2012)

Sánchez, M. Cervantes, V. y Peralta, P., Gestión de la Innovación en Pequeñas y Mediana Empresas de Barranquilla Colombia, Revista de Ciencias Sociales, XXII (2), 78-91 (2016)

Sisto, V. y López, V., Las Voces y Silencios de la Universidad Hoy: La Academia ante la Transformación de la Universidad, Psicoperspectivas, 13(1), 1-5 (2014)

Smith, H. Lovera, M. y Marín, F., Innovación Tecnológica en la Organización Empresarial. Un Análisis desde la Teoría Biológica Evolucionista, Multiciencias, 8(1), 28 - 37 (2008)

Sunkel, G. Trucco, D. y Espejo, A., La Integración de las Tecnologías Digitales en las Escuelas de América Latina y el Caribe. (En línea: https://goo.gl/gVoZDr. Acceso: 12 enero 2017) CEPAL (2014)

Tapasco, O.A. y Giraldo, J. A., Estudio Comparativo sobre Percepción y uso de las TIC entre Profesores de Universidades Públicas y Privadas, doi:10.4067/50718-520620160004000400003, Formación Universitaria (en línea), 10(2), 3-12 (2017)

Tünnermann B.C., La Educación Superior frente a los Desafíos Contemporáneos. (En línea: https://goo.gl/ZXrGXW. Acceso: 12 enero 2017) Lección Inaugural del Año Académico 2011. Universidad Centroamericana Managua, Nicaragua (2011)

Vajargah, K.F. Jahani, S. y Azadmanesh, N., Application of ICTS in Teaching and Learning at University Level: the Case of Shahid Beheshti University, The Turkish Online Journal of Educational Technology, 9(2), 33-39 (2010)

Valdés-Cuervo, A.A. Angulo-Armenta, J., Urías-Martínez, M.L. García-López y R.I. Mortis Lozoya, S.V. Necesidades de Capacitación de Docentes de Educación Básica en el Uso de las TIC, Píxel-Bit, Revista de Medios y Educación, 39(1), 211 - 223 (2011)

Zenteno-Ancira, A., Mortera Gutiérrez, F.J., Integración y Apropiación de las TIC en los Profesores y los Alumnos de Educación Media Superior, Apertura, 3(1), 1-15 (2011) 
